\title{
Considerazioni su un sistema museale diffuso delle architetture fortifícate della Valnerina
}

\section{Stefano D’Avino}

Dipartimento di Architettura Università “G. d’Annunzio” di Chieti e Pescara, Italia, e-mail: sdavino@unich.it

\begin{abstract}
The Valnerina (the valley of Nera river), in Umbria, is characterized by the diffuse distribution of military structures built between the X and XVI centuries; a set that can be traced today in an integrated route between museums and territory.

Such emergencies must be recognizable and returned to its wider historical and monumental to which they belong; of course such a program imposes a restoration project and a process of arrangement and enhancement. A new approach is the 'diffuse museum': the process of introduction to the history proceeds from the particular to the general, giving up the practice of rebuilding in a symbolic container situations, events, places. These will be sought on the ground, recovered to a historical criticism and linked to centers of interpretation and documentation; the museum becomes a reflection of a conception of heritage that proceeds by progressive selection, aiming to maintain a critical matter and the history of the monuments; an integrated system of tools that perform different functions and integrated. Such a museum will also feature a major component of virtuality that allows users to provide the context information that facilitate the understanding of a historical artifact or a work; the purpose is not to recreate 'original digital' with the aim of preserving the uniqueness of perishable monuments but to determine a network of logical relations, between chronological element, multiplicity and contexts (environmental, historical and cultural); especially by comparison with monuments, testimonies and documents congruent seats or located elsewhere. Another object is to express the possibility of analysis offered by the perception, permitted by digital reality, to observe details otherwise normally invisible.
\end{abstract}

Keywords: military structures, heritage, context, diffuse museum.

\section{Introduzione}

Terra di passaggio, posta da sempre fra confini di entità politiche forti, la valle del fiume Nera, in Umbria, mostra ancora oggi con le sue numerose architetture difensive i segni delle sue travagliate vicende storico-politiche.

Una storia di aspre offese e strenue difese testimoniata dalla distribuzione di strutture militari edificate nel corso dei secoli a presidio dei borghi e del territorio; avvenimenti che possono essere oggi ripercorsi in un itinerario affascinante che contempla tutta l'area, attivando una stretta l'integrazione fra musei e territorio; in particolare, la regione mostra straordinari esempi di quella singolare tipologia di architettura difensiva propria dell'età rinascimentale, la 'rocca', presidio di piccole guarnigioni di armati, che qui individua un esemplare laboratorio.

Va sottolineato come nel nostro Paese tale partecipazione al comune obiettivo della conoscenza storica si mostri particolarmente attiva, sebbene talvolta si evidenzi in misura meno eclatante; non a caso André Chastel ha definito l'Italia “il luogo per eccellenza del 
museo naturale", un grande 'museo a cielo aperto; integrazione fra differenti ambiti che, altresì, è caratteristica di tutto il patrimonio culturale, non solo di quello architettonico.

In questi ultimi anni, in Umbria, molto è stato fatto per delineare una rete museale a scala regionale: un sistema capace di porre all'attenzione tutte le emergenze, più o meno leggibili, incorporate nel tessuto urbano ovvero disseminate nel paesaggio; paesaggio che, peraltro, è di per se stesso parte di tale patrimonio diffuso.

Ma tali documenti, tracciati lungo i nostri percorsi quotidiani devono essere riconoscibili e leggibili anche in rapporto ai luoghi in cui sono collocati; vanno cioè restituite ai più ampi contesti storici e monumentali ai quali appartengono. Va detto, infatti, come questa regione vanti un patrimonio diversificato, costituito non solo dalle testimonianze più alte della creatività artistica e intellettuale del passato, ma anche di quei luoghi, spesso minori, che racchiudono, ciò nonostante, importanti tracce della nostra storia. Naturalmente un tale programma impone un preventivo progetto per il loro restauro e conservazione, ed un successivo processo di allestimento e valorizzazione; essi possono in tal modo divenire testimoni della storia e del patrimonio artistico territoriale e suscitatori di viva attenzione.

\section{2. il 'museo diffuso'}

Recentemente ci si è interrogati intorno all'eventualità di un diverso e nuovo approccio al patrimonio architettonico: il 'museo diffuso', ovvero l'idea che il territorio possa considerarsi alla stregua di un immenso deposito di tracce del passato che occorre riportare alla luce ed interpretare nel loro significato specifico; nonché nelle loro relazioni con il contesto locale e con la storia in generale.

L'espressione 'museo diffuso' definisce un concetto che, almeno in Italia, è relativamente recente; nato per esprimere lo stretto rapporto con il territorio che naturalmente caratterizza i musei, poi rielaborato sul finire degli anni Novanta, il termine prefigura una inversione rispetto al processo critico-cognitivo di introduzione alla storia offerto nei musei tradizionali; esso procede dal particolare al generale, rinunciando a qualsivoglia processo di mimesi, cioè alla pratica di ricostruire in un contenitore simbolico situazioni, eventi, luoghi. Questi saranno, piuttosto, ricercati sul territorio, recuperati ad una critica storica e collegati a centri d'interpretazione e di documentazione, attraverso un approccio che possiamo definire 'decentrato'; il museo diviene così il riflesso di una concezione del patrimonio che procede per progressiva selezione, mirante a conservare criticamente la materia e la storia dei monumenti.

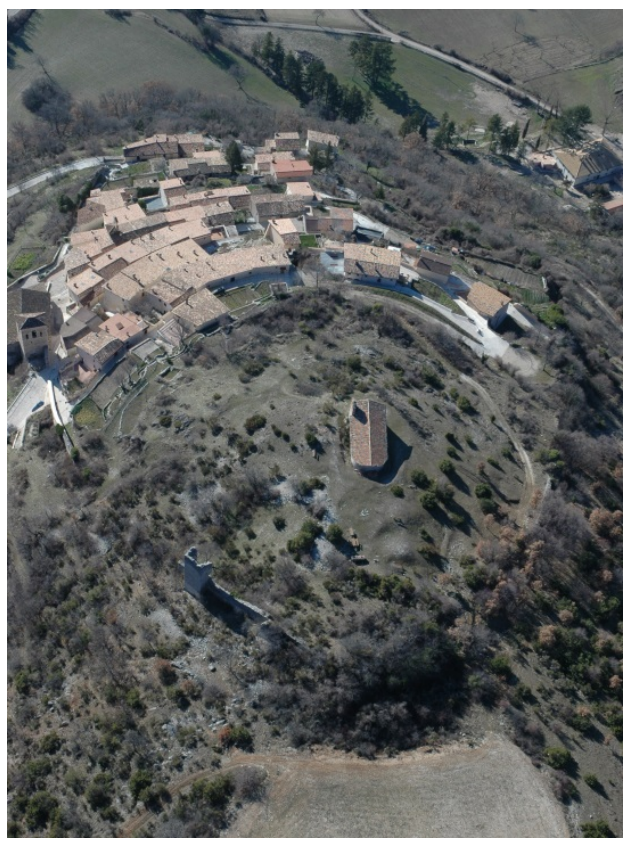

Fig. 1 - Resti della fortificazione di Campi.

Esso parte dall'assunto che una mediazione efficace dell'interesse di un pubblico indifferenziato per la storia può essere costituita dall'esplorazione del territorio in cui vive e dal successivo 'riconoscimento' dei segni (volontari, ma anche involontari, secondo la efficace definizione di Riegl) del passato; si cerca di sollecitare, cioè, la fascinazione che esercita il viaggio nello spazio e nel tempo, portando in luce la storia di luoghi, persone, cose poco note o note solo nella loro funzione e parvenza contingente, secondo un approccio che fa leva 
sulla relazione fra emozioni soggettive e ambiente.

Le contiguità e le possibili sinergie fra questa disciplina e una museologia volta al recupero della dimensione storica attraverso i 'luoghi della memoria', ma soprattutto la 'memoria dei luoghi' sono evidenti, benché sostanzialmente ancora inesplorate.

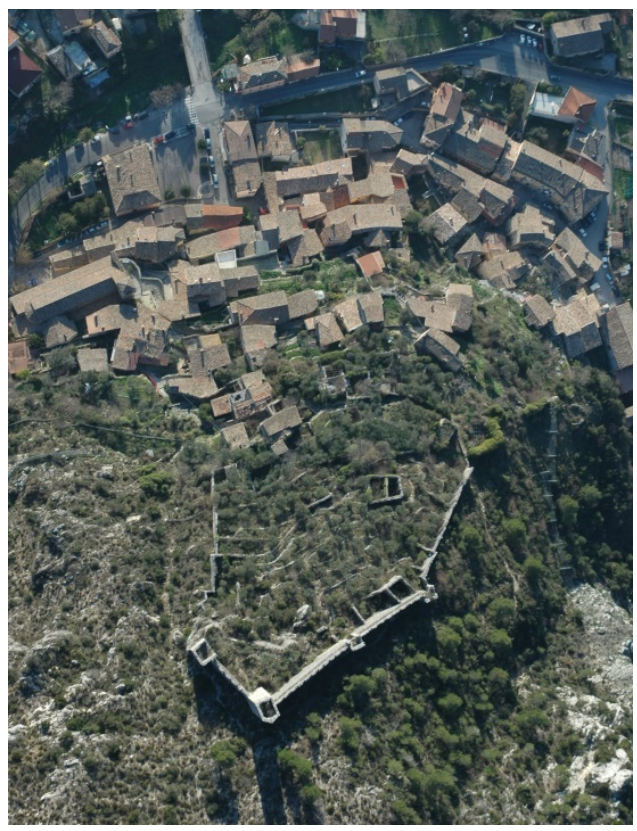

Fig. 2 - Ferentillo (Terni). Castello di Precetto.

La specificità del museo diffuso consiste nel suo essere un sistema articolato di strumenti che svolgono funzioni diverse ed integrate. E' stato osservato come, in realtà, tutti i musei locali che illustrano aspetti del territorio dovrebbero aumentare questo loro valore contestualizzante, creando itinerari di visita e supporti didattici che rimandino il visitatore ad una conoscenza più attenta del luogo; la scelta di uscire dal museo diviene una risposta alla curiosità che tuttavia sussiste da parte del pubblico nei confronti delle vicende storiche che il museo si propone di affrontare; un processo cognitivo ricco di continui rimandi critici.

Progettare, dunque, un 'Museo diffuso delle architetture fortificate' significa fissare un metodo di fruizione dell'intero territorio; ciò può realizzarsi attraverso l'attivazione di itinerari che privilegino mete normalmente quasi ignorate dai flussi turistici, o itinerari che diano unità a più elementi coerenti fra loro, diffusi nel territorio e concorrenti ad un medesimo indirizzo storicocritico; tale percorso può avvalersi di pubblicazioni come pure di prodotti audiovisivi e multimediali innovativi.

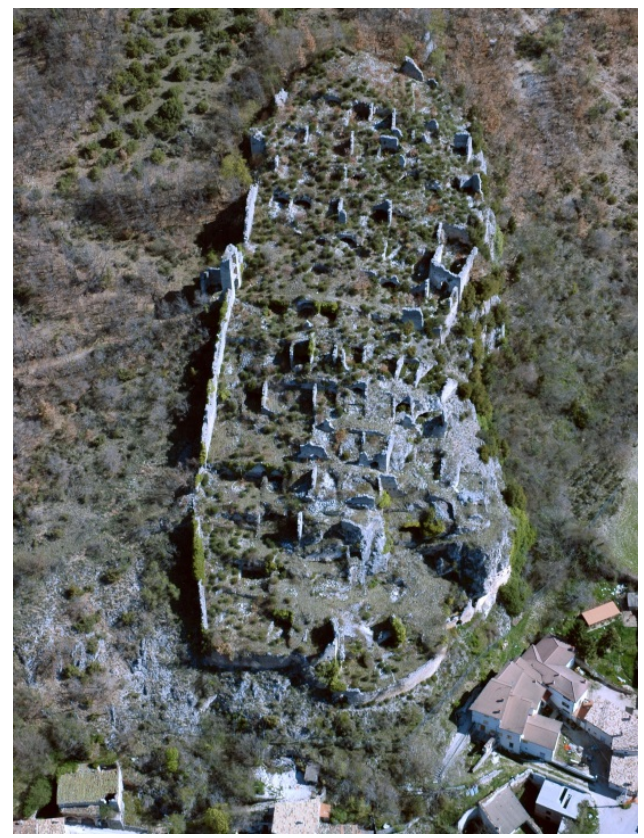

Fig. 3 - Ruderi di Castelfranco d'Ancarano.

Due sono i componenti primari del museo diffuso: un sistema di percorsi che colleghino le emergenze ed i luoghi di interesse scientifico e culturale; un polo di riferimento dove concentrare l'essenza specifica della struttura museale (servizi al pubblico, luogo di raccolta e di documentazione, ...) ideato, anche, come laboratorio permanente per la salvaguardia e valorizzazione del paesaggio (un 'punto di coordinamento' che articolerà a rete le varie componenti, ovvero i siti distribuiti nei territori comunali.

Si definirà pertanto un museo caratterizzato da un'importante componente di virtualità che consenta, affiancandosi ai compiti didattici ed espositivi istituzionalmente svolti dalle strutture museali tradizionali, di fornire agli utenti quelle 
informazioni di contesto che facilitano la comprensione storica di un reperto o di un'opera.

Un programma multimediale con un'ampia collezione di risorse digitali, che potrebbe agevolmente essere installato su un navigatore portatile, permetterà al visitatore di individuare agevolmente i poli di interesse, porli fra loro in rapporto e trarre molteplici note criticoesplicative; coinvolgendolo, nel contempo, nella lettura dell'opera, stimolandolo ad esplorarne la conoscenza, attivando i collegamenti tematici ed intellettuali necessari per coglierne il portato e collocarla nel suo specifico ambito.

Il riferimento alla sfera virtuale della musealizzazione appare pertinente: lo scopo non è quello di ricreare 'originali digitali' ovvero 'doppi originali' con l'esclusivo fine di conservare la deperibile singolarità dei monumenti quanto, piuttosto, di determinare una rete strutturante di relazioni logiche, cronologiche fra elemento, molteplicità e contesti (ambientale, storico e culturale), particolarmente attraverso il confronto con monumenti, testimonianze e documenti congruenti posti o collocati altrove; nonché esprimere la possibilità di analisi offerta dalla percezione, consentita dalla realtà digitale, di osservare dettagli altrimenti normalmente invisibili.

\section{Conclusioni}

Attraverso l'indispensabile sinergia fra tutti i soggetti attivi nel territorio potrà al fine realizzasi questo ambizioso progetto di recupero, protezione e salvaguardia, allestimento e valorizzazione dei monumenti; testimonianze che possono divenire vetrine della storia e del patrimonio archeologico, architettonico ed artistico territoriale, attrattori di attenzione, suscitatori di curiosità; nonché sollecitare a compiere più ampie esplorazioni e ricerche d'informazioni. A partire dell'analisi del territorio, inteso come deposito di tracce del passato, saremmo in tal modo in grado di riconoscere e decifrare tali testimonianze architettoniche, comprendendo il loro significato nel contesto locale e stabilendo un rapporto con la storia.

Proprio in quanto sovente si tratta di luoghi ed edifici che gli avvenimenti successivi hanno cancellato, trasformato o convertito ad altre funzioni, riscoprire questi luoghi - spesso dimenticati, talvolta sconosciuti - può aiutare a rileggere le vicende storiche della comunità della quale si fa parte, ricostruendone, attraverso la memoria, l'identità. Si tratta, in altre parole, di interrogare la memoria dei luoghi.

Dal punto di vista del pubblico, l'impatto con la dimensione diacronica di luoghi conosciuti solo nella dimensione del presente, l'approccio cognitivo offerto dal sistema museale diffuso può suscitare un elevato interesse per la storia, innescando un collegamento critico fra il presente e il passato ignoto, fra il particolare ed il generale e mostrando cose tangibili e visibili in una dimensione temporale che appare ancora del tutto inesplorata.

\section{References}

Chastel A., (1980), L’Italia, museo dei musei, Milano.

D’Avino S., (2009), "Sancta dicta sunt et quotidie debent reparari. Architetture difensive in Valnerina”, in Contributi, n.s., 9, Carsa edizioni, Pescara.

D’Avino S., (2014), “Comprendre, c'est traduire. Riflessioni sul complesso rapporto fra archeologia e conservazione”, in Caiete ARA 5, atti del $14^{\circ}$ Symposium ARA Architecture. Restoration. Archaeology, Bucarest 18-20/4/2013, Bucarest, pp. 179-188.

D’Avino S., (2014), “L’immagine possibile. L'esercizio della virtualità nei processi di musealizzazione”, in Messico Italia Restauro, a cura di Marcello D’Anselmo, Roma, pp. 181-194.

Daverio Ph., Trapani V., (2013), La sostenibilità del museo e il museo verde, Milano.

Passaro A., (2003), "Paesaggio come documento", in Esperienze innovative per la configurazione del paesaggio rurale , Napoli.

Vitale G., (2010), Il museo visibile, Milano. 